Received: 28 February 2017

Accepted: 30 August 2017

Published online: 14 September 2017
NTIFIC REP

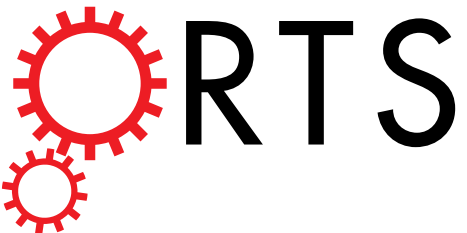

Corrected: Author Correction

\title{
OPEN TGF- $\beta 1$-induced miR-503 controls cell growth and apoptosis by targeting PDCD4 in glioblastoma cells
}

\begin{abstract}
Pin Guo ${ }^{1}$, Yanan $\mathrm{Yu}^{2}$, Huanting $\mathrm{Li}^{1}$, Daoxiang Zhang ${ }^{3}$, Anjing Gong ${ }^{1}$, Shifang $\mathrm{Li}^{1}$, Wei Liu ${ }^{1}$, Lei Cheng ${ }^{1}$, Yongming Qiu $^{4}$, Weicheng Yao ${ }^{1}$, Luo Li $^{5}$ \& Yugong Feng ${ }^{1}$
\end{abstract}

Aberrant expression of microRNAs hae been shown to be closely associated with glioblastoma cell proliferation, apoptosis and drug resistance. However, mechanisms underlying the role of mcroRNAs in glioblastoma cell growth and apoptosis are not fully understood. In this study, we report that miR-503 is overexpressed in glioblastoma tissue compared with normal human brain tissue. Mechanistically, miR- 503 can be induced by TGF- $\beta 1$ at the transcriptional level by binding the smad $2 / 3$ binding elements in the promoter. Ectopic overexpression of miR-503 promotes cell growth and inhibits apoptosis by targeting PDCD4. In contrast, inhibition of miR-503 reduces cell growth. Furthermore, miR-503 inhibitor augments the growth inhibitory effect of temozolomide in glioblastoma cells. These results establish miR-503 as a promising molecular target for glioblastoma therapy.

Glioblastoma, which accounts for approximately $80 \%$ of primary malignant brain tumors, is among the most prognostically discouraging adult neoplasias ${ }^{1}$. The annual incidence of glioblastoma is about 3-8 per 10 million people $^{2}$. The median survival of glioblastoma patients is less than one year from diagnosis, which is lower than that for patients with any other kind of tumor ${ }^{3}$. Current therapies for glioblastoma, including radiotherapy, surgery and chemotherapy, have not been successful due to the highly aggressive nature of the tumor ${ }^{1,2}$. Therefore, there is an urgent demand to understand the molecular mechanisms controlling glioblastoma progression and manifestation.

MicroRNAs (miRNAs) are a class of 18-22 bp non-coding RNAs that play key roles in cell proliferation, apoptosis and differentiation ${ }^{4}$. MicroRNAs can act as both oncogenes and tumor suppressors by negatively regulating mRNA through either translational repression or mRNA degradation ${ }^{5}$. In recent years, the aberrant expression of many microRNAs has been reported to be associated with progression of various cancers. These aberrantly expressed microRNAs have been identified as novel potential targets for cancer therapy ${ }^{6}$. Therefore, exploring the role and the mechanism of microRNAs dysregulation in cancer is essential for diagnosis and therapy.

Aberrant expression of miR-503 has been shown in several types of cancers and appears to be significantly associated with clinical outcome in patients. For example, downregulation of microRNA-503 expression level predicates advanced cytological features and poor prognosis in patients with non-small cell lung cancer ${ }^{7}$. In prostate cancer, microRNA-503 can directly regulate RNF31 and thus suppress tumor cell proliferation and metastasis $^{8}$. Analysis of microRNA expression profiling shows that microRNA-503 regulates metastatic function in hepatocellular cancer cells ${ }^{9}$. However, microRNA-503 was found to be upregulated in esophageal cancer tissues compared to adjacent normal tissues and to promote tumor progression ${ }^{10}$. Feinmesser et al. reported that upregulation of miR-503 was the best single discriminator of malignancy ${ }^{11}$. On the basis of these previous reports, downregulation or upregulation is cancer type-specific. In glioblastoma, the role of miR-503 remains largely unknown.

${ }^{1}$ Department of Neurosurgery, the Affiliated Hospital of Qingdao University, Qingdao, China. ${ }^{2}$ Department of Gastroenterology, the Affiliated Hospital of Qingdao University, Qingdao, China. ${ }^{3}$ Division of Oncology, Department of Internal Medicine, Washington University School of Medicine, Saint Louis, MO, 63110, USA. ${ }^{4}$ Department of Neurosurgery, South Campus, Renji Hospital, Shanghai Jiao Tong University School of Medicine, Shanghai, China. ${ }^{5}$ Department of Neurology, Qingdao Municipal Hospital, Qingdao University, Qingdao, China. Pin Guo and Yanan Yu contributed equally to this work. Correspondence and requests for materials should be addressed to Y.F. (email: fengyugong001@163.com) or L.L. (email: liluodoctor@163.com) 
A

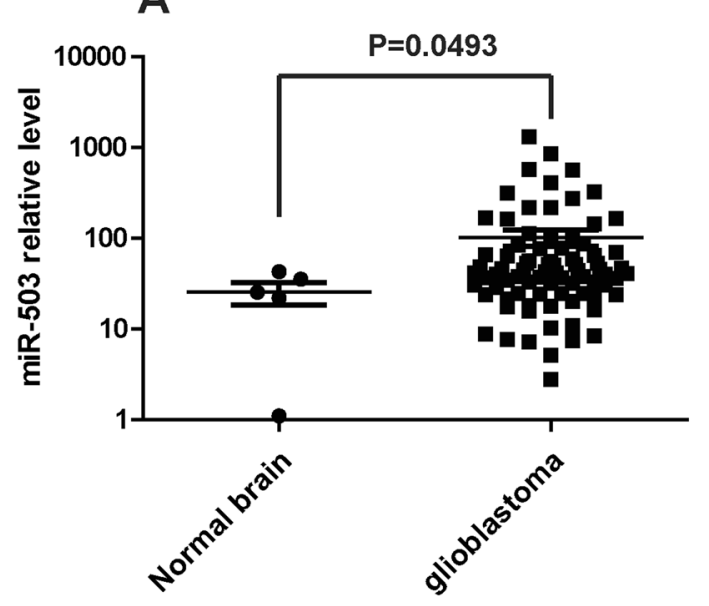

B

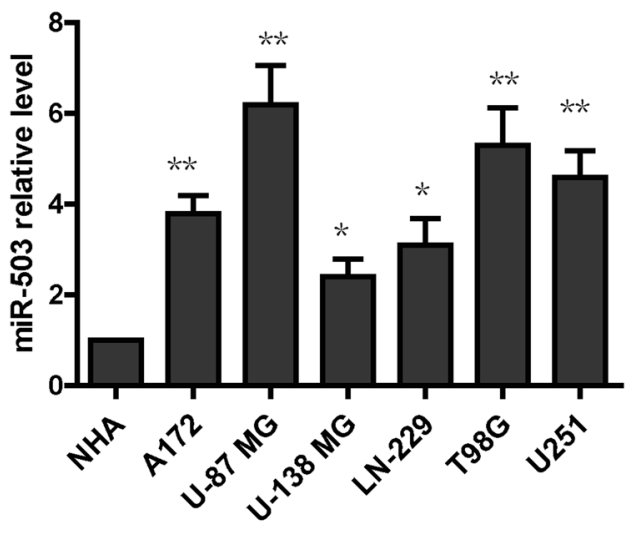

Figure 1. miR-503 is up-regulated in glioblastoma. (A) miR-503 expression was analyzed from the microRNA profiling in glioblastoma tissues and normal brain tissues. (B) The level of miR-503 was individually analyzed using quantitative PCR analysis insix glioblastoma cell lines (U251, A172, LN-229, T98G, U87MG and $\mathrm{U}-138 \mathrm{MG}$ ) and in normal human astrocytes (NHAs).

In this report, we found that miR-503 is overexpressed in human glioblastoma tissues compared with normal brain tissues, and that TGF- $\beta 1$ can induce miR-503 expression at the transcriptional level by binding the promoter. Our biological function assay showed that miR-503 enhances cell proliferation and inhibits apoptosis by targeting PDCD4. Furthermore, miR-503 inhibitor augments the growth-inhibitory effect of temozolomide in glioblastoma cells. These results establish miR-503 as a promising molecular target for glioblastoma therapy.

\section{Results}

miR-503 is up-regulated in glioblastoma. To examine the expression level of miR-503 in human glioblastoma, we analyzed the miRNA's expression profile from a previously published dataset (Gene expression omnibus accession GSE25631). The result showed that miR-503 is significantly upregulated in glioblastoma tissue compared with normal brain tissue $(\mathrm{p}<0.001)$ (Fig. 1A). To further confirm the expression level of miR-503 in glioblastoma, we performed qRT-PCR to detect the level of miR-503 in glioblastoma cell lines. The result showed that the miR-503 level is higher in the six glioblastoma cell lines (U251, A172, LN-229, T98G, U87MG and U-138MG) than in normal human astrocytes (NHAs) (Fig. 1B). In conclusion, miR-503 is overexpressed in glioblastoma.

TGF- $\beta 1$ induces the expression of miR-503 in glioblastoma cells. Previous studies have shown that miR-503 is dysregulated in several types of cancer, however, the mechanism underlying the regulation of miR503 in cancer remains unknown. Jongmin Kim et al. reported that miR-503 and miR-424, which are separated by $250 \mathrm{bp}$ on the $\mathrm{X}$ chromosome, are transcribed as a single transcript. To explore whether there is a relationship between the levels of miR-503 level and miR-424, we analyzed the global microRNA expression of the published NCI-60 cancer cell panel. We found that miR-503 and miR-424 levels have a strong correlation $(\mathrm{R} 2=0.7421)$ (Fig. 2A), which indicates that miR-503 and miR-424 may be governed by the same mechanism. Zhang et al. reported that miR-424 can be induced by TGF- $\beta 1$ in normal fibroblasts. To explore whether TGF- $\beta 1$ induces miR-503 expression, we treated glioblastoma cells with different TGF- $\beta 1$ doses, ranging from $2 \mathrm{ng} / \mathrm{ml}$ to $16 \mathrm{ng} / \mathrm{ml}$, and then performed qRT-PCR to determine miR-503 levels. The result showed that expression of mature miR-503 increased with the TGF- $\beta 1$ dose increase (Fig. $2 \mathrm{~B}$ ). To further confirm the effect of TGF- $\beta 1$ on miR-503 expression, TGF- $\beta 1$ was administered to glioblastoma cells at final concentration of $8 \mathrm{ng} / \mathrm{ml}$ and qRT-PCR was performed at different time points $(0,48,72$, and 96 hours). qRT-PCR showed that miR-503 expression was enhanced under TGF- $\beta 1$ treatment, and was highest at 72 hours in three glioblastoma cell lines and at 96 hours in T98G cells (Fig. 2 C). As smad $2 / 3$ is the key mediator of the canonical TGF- $\beta 1$ pathway, we explored whether endogenous need of TGF- $\beta 1$ pathway for the miR-503 regulation in glioblastoma cells. To investigate this, we examined the response of glioblastoma cells to TGF- $\beta 1$ signaling. Western blot results showed the phosphorylation of $\operatorname{smad} 2 / 3$ is markedly enhanced in four glioblastoma cell lines under TGF- $\beta 1$ treatment for 30 minutes (Fig. $2 \mathrm{D}$ ). To further confirm this, we detected the PAI- 1 and Smad 7 mRNA level in glioblastoma cells treated with TGF- $\beta 1$, the data showed that PAI-1 mRNA was increased significantly in four cells treated with TGFb1 for $48 \mathrm{~h}$, however Smad7 mRNA was not enhanced as highly and significantly as PAI-1 mRNA (Fig. 1S). Next we treated the cells with TGF- $\beta 1$ alone or with K02288 or SB-431542, which are selective inhibitors involved in BMP signaling and TGF- $\beta$ signaling, respectively. qRT-PCR showed that miR-503 expression was suppressed by SB-431542 inhibitor, but not by K02288 inhibitor under TGF- $\beta 1$ treatment. (Fig. $2 \mathrm{E}$ ). Taken together, these data indicate that TGF- $\beta 1$ regulates the expression of miR-503 in glioblastoma via the $\operatorname{smad} 2 / 3$ pathway. 
A

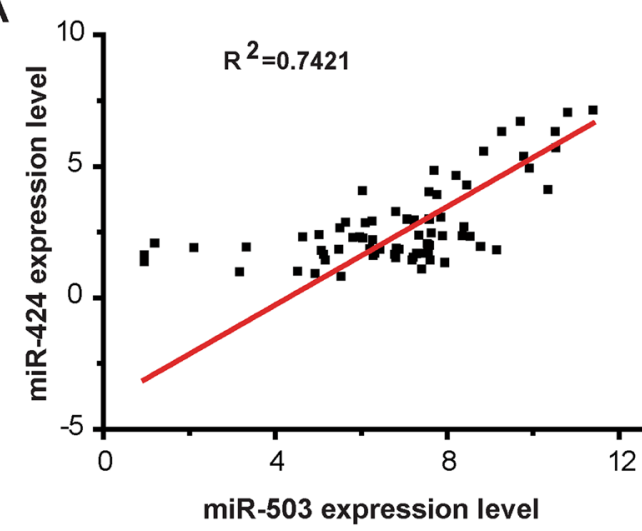

C

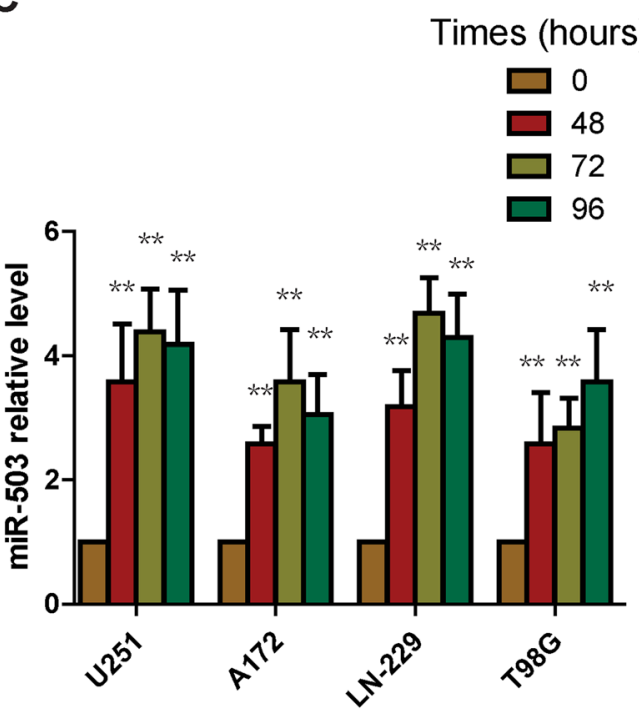

B

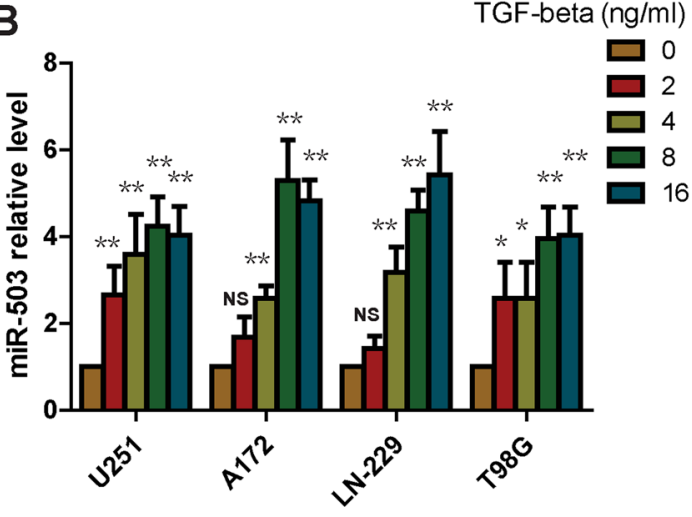

$E$
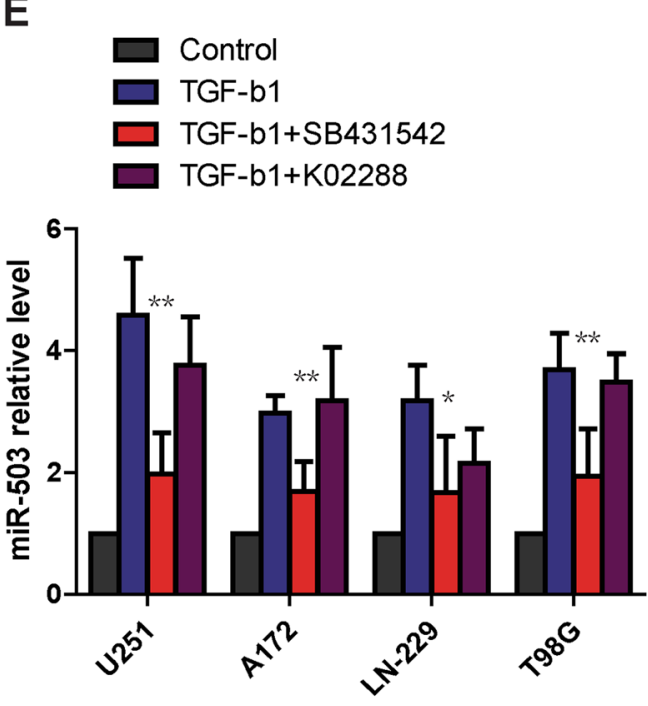

D
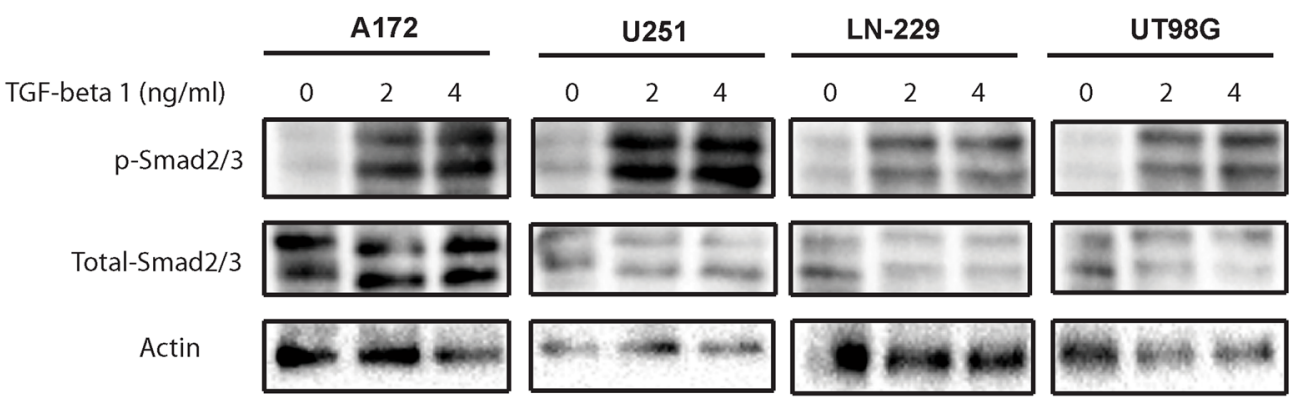

Figure 2. TGF- $\beta 1$ induces the expression of miR-503 in glioblastoma cells. (A) Correlation analysis of miR-503 level and miR-424 level in the NCI 60 cancer cell panel using Pearson correlation $\left(R^{2}=0.7421\right)$. (B) Quantitative PCR analysis of miR-503 expression in U251, A172, LN-229 and T98G cells treated with TGF- $\beta 1$ at different doses for 48 h. (C) Quantitative PCR analysis of miR-503 expression in U251, A172, LN-229 and T98G cells treated with $8 \mathrm{ng} / \mathrm{ml}$ TGF- $\beta 1$ at different time points. (D) Western blot analysis showing the phosphorylation of Smad2/3 in U251, A172,LN-229 and T98G cells treated with TGF- $\beta 1$ at different doses for 30 min. (E) Quantitative PCR analysis of miR-503 expression in U251, A172, LN-229 and T98G cells treated with TGF- $\beta 1$ alone or with SB-431542 or K02288 for $48 \mathrm{~h}$.

TGF- $\beta 1$ induces the expression of miR-503 at the level of transcription. TGF- $\beta 1$ signaling modulates downstream gene expression at the transcriptional level. This is mediated through the transcription factors $\operatorname{smad} 2$, smad 3 and smad $4^{12,13}$. To explore whether TGF- $\beta 1$ stimulation could induce miR-503 expression at the transcriptional level, we detected the pri-miR-503 level in glioblastoma cells under TGF- $\beta 1$ treatment. qRT-PCR showed that TGF- $\beta 1$ administration strongly induced pri-miR-503 expression (Fig. 3A). To further identify how TGF- $\beta 1$ regulates miR-503 expression, we analyzed the $2500 \mathrm{bp}$ region upstream of pri-miR-503 using five pairs 

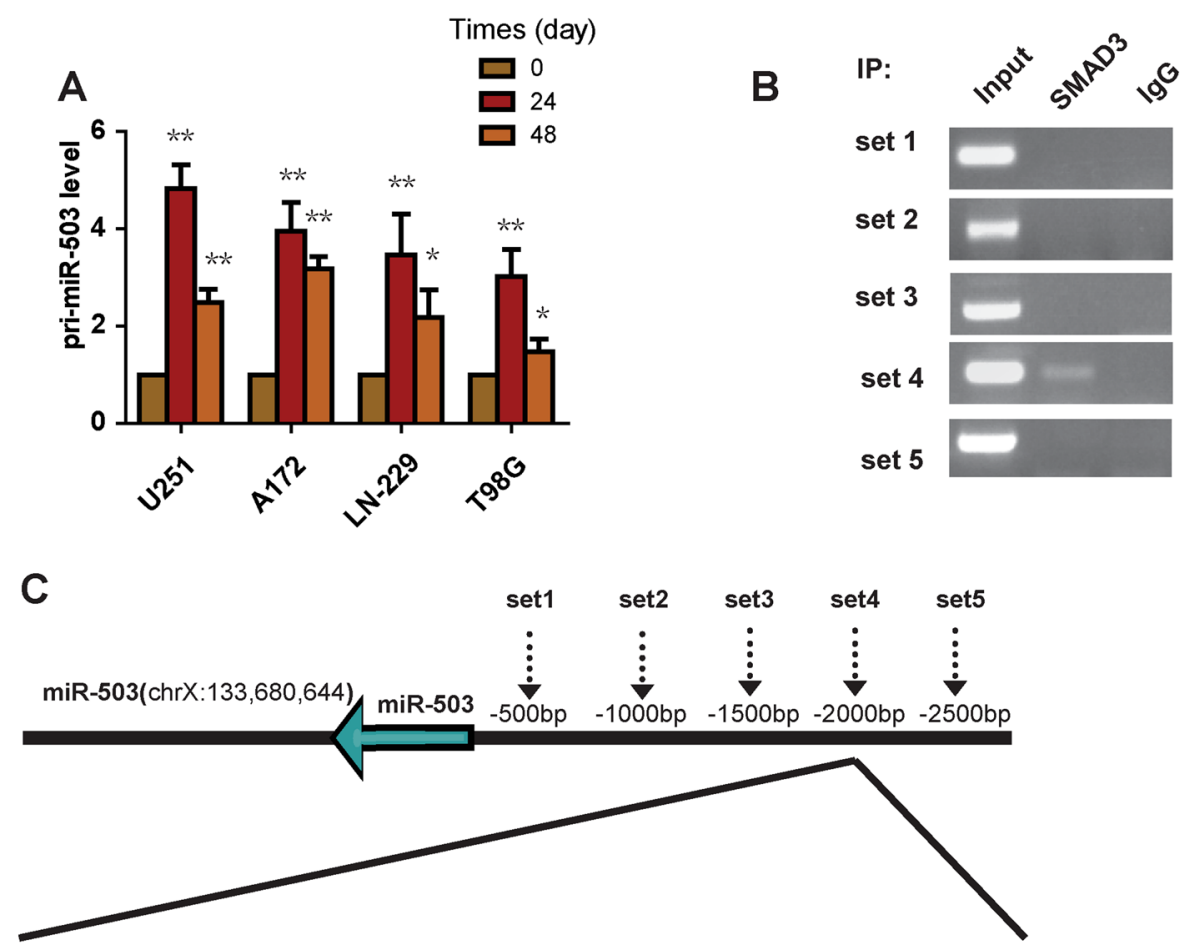

$(-1979 \mathrm{bp})$ ggggacgaag gcatatgcaa aattaaaaga caaaacgttg tagagtatga acagaggaag
aggcgtattc ttggctctt aaatcatta gccaaacaca ttgcagttca gaagggggtg
gggctatggc aactcgaaag tatcgattct ttgtctattg tatcagtgaa tgaccctctc
ctgaagtgac aagacgccce ttgtaatct ctttcgtaa g taaattca gtctaagggg (-1678bp)

$-1979 b p$

$-1678 \mathrm{bp}$

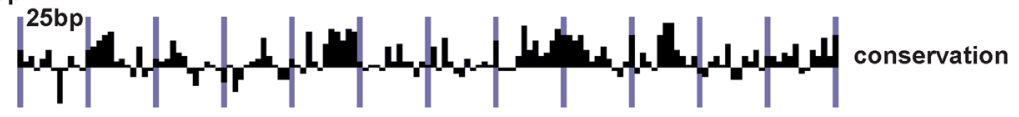

D

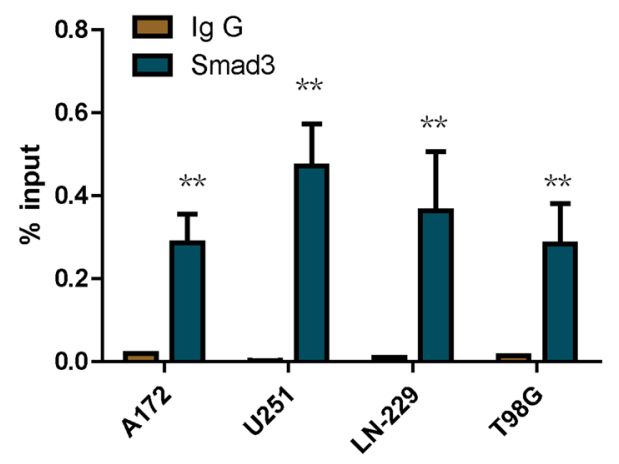

E

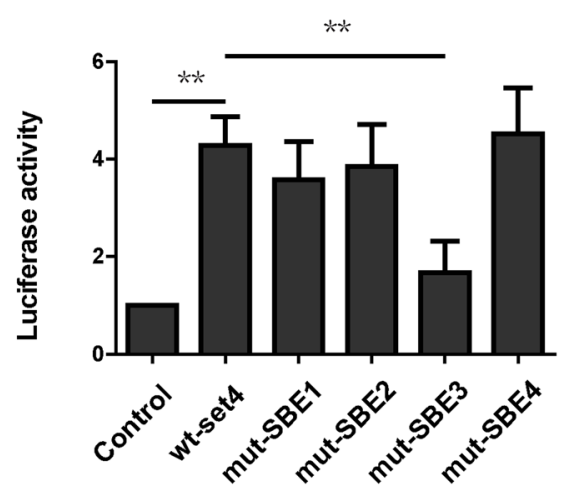

Figure 3. TGF- $\beta 1$ induces the expression of miR-503 at the transcriptional level. (A) Pri-miR-503 expression was analyzed using quantitative PCR in U251, A172, LN-229 and T98G cells treated with TGF- $\beta 1$. (B) ChIPPCR amplification products using primers against the miR-503 promoter region were separated by agarose gel electrophoresis. (C) Evolutionary conservation of the set 4 region in miR-503 promoter was shown. (D) qChIP analysis showing the enrichment of Smad2/3 in set 4 in U251, A172, LN-229 and T98G cells. (E) The luciferase reporter assay showing that mut-SBE3 significantly inhibits the increased luciferase activity induced by TGF- $\beta 1$.

of primers to create a library of five $500 \mathrm{bp}$ regions spanning the miR-503 promoter (Fig. $3 \mathrm{C}$ ). We tested each of these regions for association with SMADs. We performed ChIP experiments for smad2/3. Enriched DNA from immunoprecipitates was used for PCR and RT-PCR. Only the set 4 region of the promoter was found to be 
A

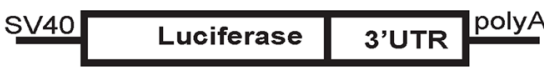

Wt 3'UTR 5' uaggggguaaaguuaaGCUGCUa 3'

miR-503 3' bgacgucuugacaagggCGACGAu 5'

miR-424 3' aaguuuuguacuuaaCGACGAc 5'

mut 3'UTR 5' uaggggguaaaguuaaGCUGCUa 3'

C

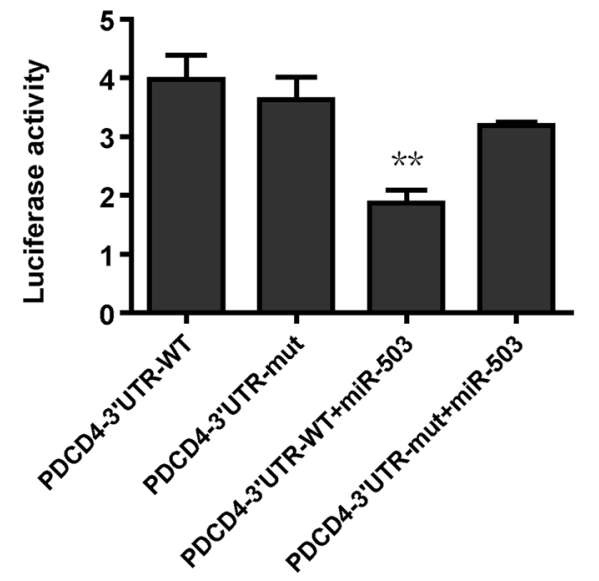

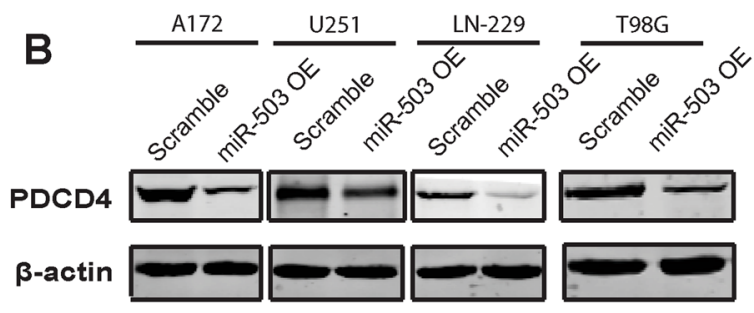

Scramble
miR-503 mimics
miR-503 inhibitor

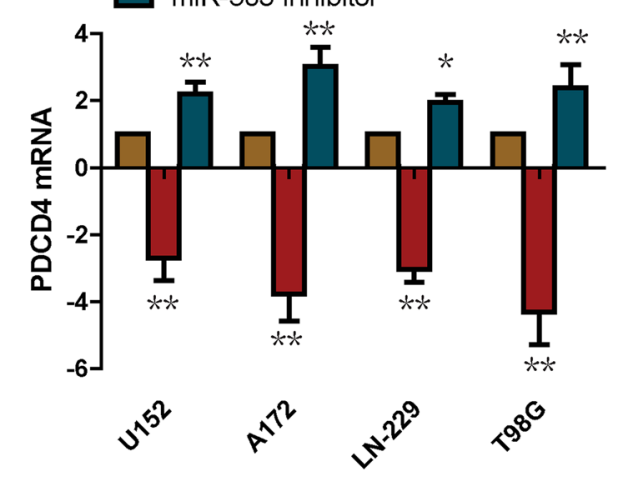

Figure 4. miR-503 suppresses PDCD4 expression. (A) The $3^{\prime}$ UTR sequence of PDCD4 is predicted as a potential binding site for miR-503. The red nucleotides are mutated to their complementary nucleotides. The blue nucleotides are the seed region of miR-424 and miR-503. (B) Western blot results showing the level of PDCD4 protein in U251, A172 and LN-229 cells transfected with miR-503 mimics. (C) Luciferase reporter assay showing the luciferase activity in cells transfected with the wild type $3^{\prime}$ UTR or the mutant $3^{\prime}$ UTR with miR-503 mimics. (D) Quantitative PCR analysis of the PDCD4 mRNA level in U251, A172, LN-229 and T98G cells transfected with miR-503 inhibitor or miR-503 mimics.

associated with enrichment of smad2/3 (Fig. 3B). No enrichment was detected with an isotype-matched IgG antibody (Fig. 3D). In the set 4 region, we found 4 copies of the sequence GTCT or AGAC (smad-binding elements, SBEs) (Fig. 3C), which can be bound by the smad3/smad 4 complex. To further explore whether smad $2 / 3$ binds the SBEs, we cloned the set 4 region into the pGL3 promoter and mutated each SBE individually. HEK293T cells were transfected with the control plasmid, wild type set 4 region plasmid or mutant plasmid. In the luciferase reporter assay, wild-type set 4 region plasmid significantly enhanced the luciferase activity (Fig. 3E). A mutation in SBE 3 led to a significant decrease in luciferase activity, however, mutation in SBE1, SBE2, SBE4 showed no decreased luciferase activity compared with the wild-type set 4 region plasmid (Fig. 3E). We also found that the SBE4 is highly conserved in the promoter region (Fig. 3C). In conclusion, Smad2/3 binds to the SBE3 in the set 4 region of the pri-miR-503 promoter and directly activates its transcription.

miR-503 suppresses PDCD4 expression. To further detect the mechanism by which miR-503 increases glioblastoma cell growth and suppresses apoptosis, we compared the seed region sequences of miR-503 and miR424. These two miRNAs share substantial sequence identity in their seed region (Fig. 4A) ${ }^{14}$. miR-424 was reported to promote tumor resistance to apoptosis by targeting the expression of PDCD4, which is a well-established tumor suppressor, known to regulate cell growth and apoptosis ${ }^{15}$. On the basis of these findings, we hypothesized that PDCD4 is a potential target of miR-503. We detected the level of PDCD4 protein using western blot in cells overexpressing miR-503. The data showed that overexpression of miR-503 dramatically decreased the level of PDCD4 protein (Fig. 4B). Moreover, we analyzed the level of PDCD4 mRNA using qRT-PCR in glioblastoma cells transfected with miR-503 inhibitor or mimics. The data showed that miR-503 inhibitor increased the level of PDCD4 mRNA. In contrast, miR-503 mimics reduced the PDCD4 mRNA level (Fig. 4D). Furthermore, to confirm whether PDCD4 is indeed the target of miR-503, we cloned the $3^{\prime}$ UTR of PDCD4 into the dual-luciferase UTR vector and performed the luciferase reporter assay (Fig. 4C). The data showed that luciferase activity was significantly decreased in cells cotransfected with miR-503 mimics and 3' UTR-wild-type, but not in cells cotransfected with miR-503 mimics and 3' UTR-mutant. Taken together, these data suggest that miR-503 downregulates the expression of PDCD4.

miR-503 increases glioblastoma cell proliferation and inhibits cell apoptosis. To examine the biological role of miR-503 in glioblastoma cells, we transfected glioblastoma cells with miR-503 inhibitor or 
A
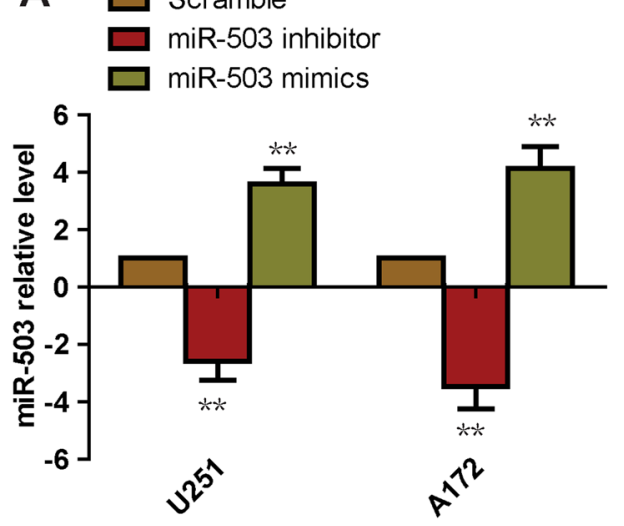

C

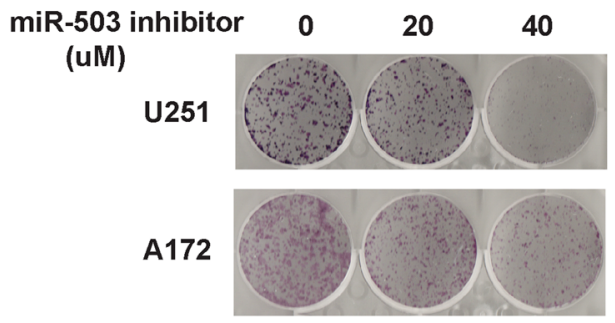

B

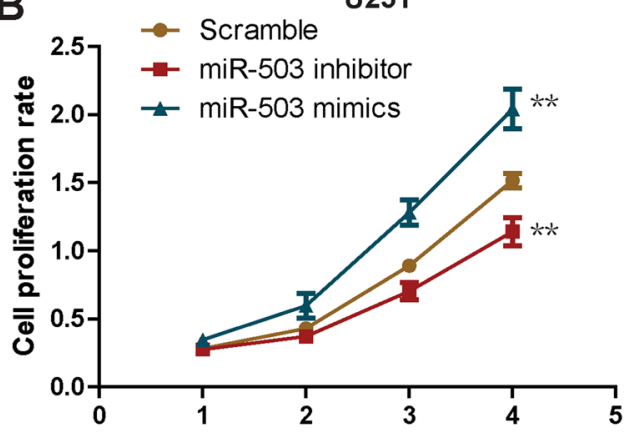

D

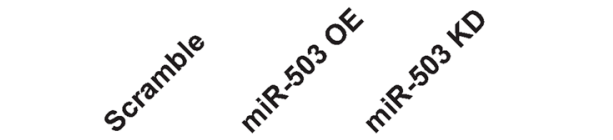

U251

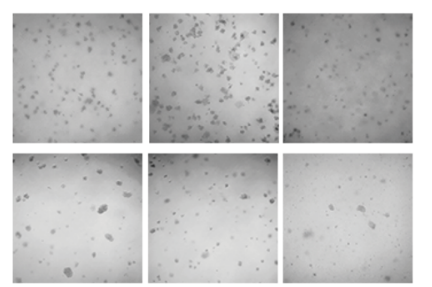

E

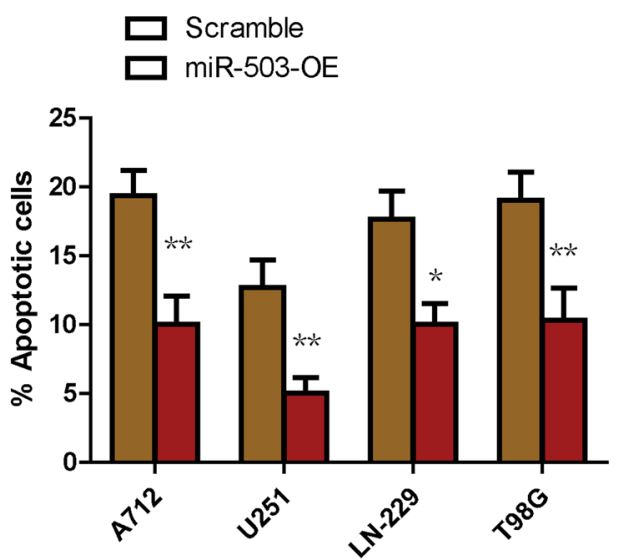

Figure 5. miR-503 increases glioblastoma cell proliferation and inhibits cell apoptosis. (A) The knockdown or overexpression efficacy of miR-503 was analyzed using quantitative PCR in U251 and A172 transfected with miR-503 inhibitor or miR-503 mimics. (B) The cell proliferation rate was measured with the Alarma Blue assay in cells transfected with miR-503 inhibitor or miR-503 mimics. (C) Colony formation assay of cells transfected with miR-503 inhibitors. (D) Anchorage growth of cells transfected with miR-503 mimics in soft agar. (E) Flow cytometry analysis of the annexin-V positive populations of cells treated with miR-503 mimics..

mimic to knockdown or overexpress the endogenous miR-503, respectively. We used qRT-PCR to demonstrate that miR-503 was indeed dramatically decreased or enhanced as expected (Fig. 5A). We seeded the miR-503 knockdown or overexpression cells in 96 well plates and cultured them to different time points for the Alarma Blue cell growth assay. The results showed that overexpression of miR-503 enhanced cell proliferation, and in contrast, knockdown of miR-503 suppressed glioblastoma cell growth (Fig. 5B). We next tested the effect of miR503 inhibitor on colony formation in vitro. We found that miR-503 inhibitor strongly repressed colony formation in a dose dependent manner (Fig. 5C). To further confirm these findings, we then performed the soft agar assay, which is widely accepted as the most stringent assay for transformed growth. The result showed that overexpression of miR-503 enhanced anchorage-independent growth, as expected, and knockdown of miR-503 suppressed anchorage-independent growth (Fig. 5D), indicating an essential role of miR-503 in cell growth. We next sought to explore the role of miR-503 in apoptosis. We stained cells overexpressing miR-503 with annexin V and PI for flow cytometry analysis. The data showed a lower percentage of annexin V positive cells in the miR-503 

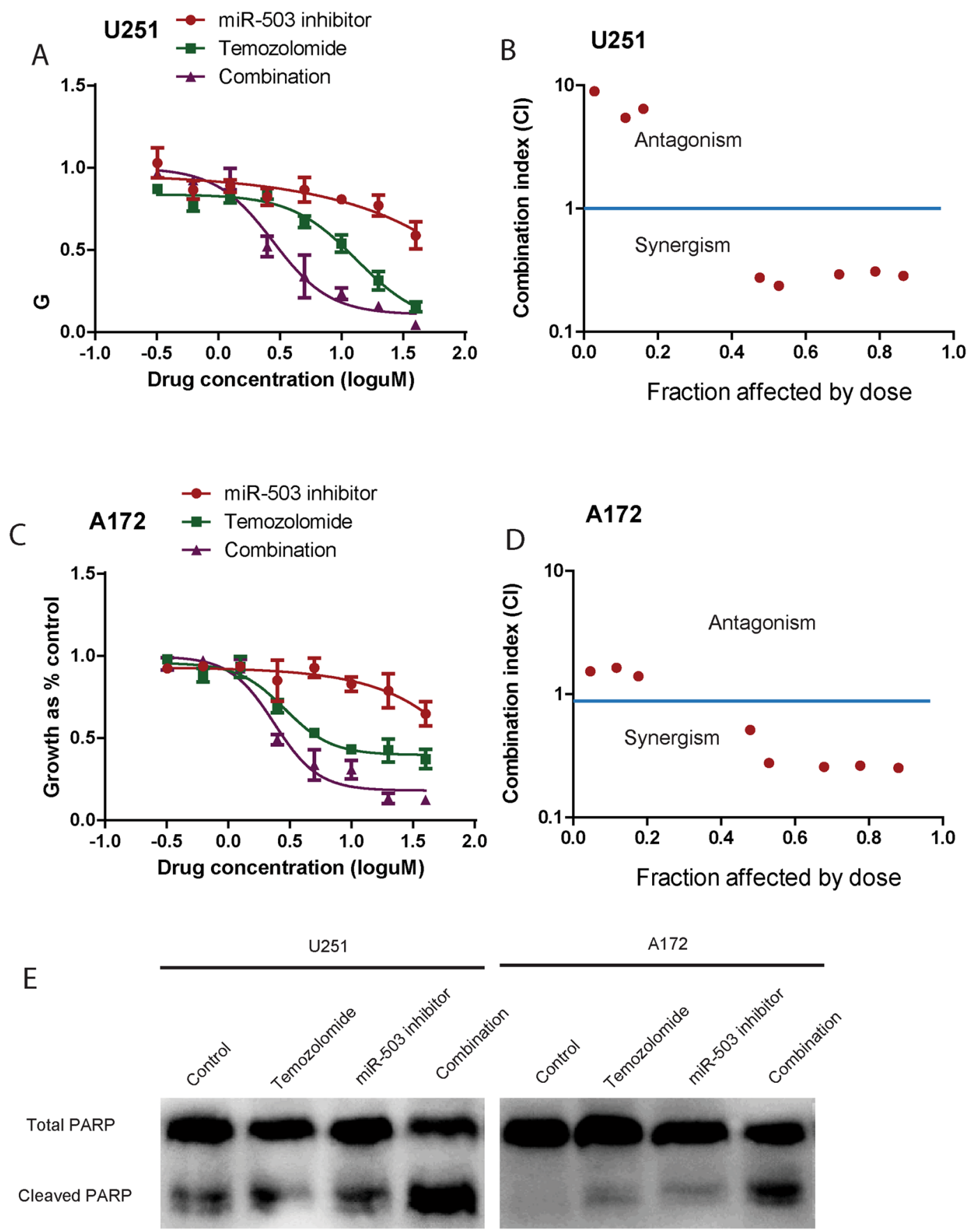

Figure 6. Growth inhibition induced by miR-503 inhibitor and temozolomide, alone and in combination. (A) and (C). U251 and A172 cells were treated with various combinations of miR-503 inhibitor and temozolomide for $72 \mathrm{~h}$. The growth-inhibitory effect was measured by the Alarma Blue assay. (B) and (D) Median effect analysis conducted using Calcusyn software showing interaction between miR-503 inhibitor and temozolomide in U251 and A172 cells. The combination effect was plotted as CI against the fraction affected. When the $\mathrm{CI}$ value is $<1$, the interaction between two drugs is considered synergistic, when $\mathrm{CI}=1$, the interaction is additive, and when $\mathrm{CI}>1$, the interaction is antagonistic. (E) The total PARP and cleaved PARP were detected using western blot analysis in U215 and A172 cells, which were transfect with a scrambled oligo or miR-503 inhibitor. The final concentration of temozolomide was $10 \mu \mathrm{M}$.

overexpression group than in the scramble cells (Fig. 5E), suggesting miR-503 overexpression suppresses glioblastoma cell apoptosis.

miR-503 inhibitor and temozolomide inhibit glioblastoma cell growth alone and in combination. To translate the above findings, we next examined the effect of combined miR-503 inhibitor and the first-line glioblastoma multiforme treatment, temozolomide ${ }^{16}$, on glioblastoma cells over $72 \mathrm{~h}$. The miR-503 inhibitor 
and temozolomide were used alone at $0.31 \mathrm{uM}, 0.62 \mathrm{uM}, 1.25 \mathrm{uM}, 2.5 \mathrm{uM}, 5 \mathrm{uM}, 10 \mathrm{uM}, 20 \mathrm{uM}, 40 \mathrm{uM}$, and in combination at equipotent concentrations at the same ratios. The Alarma Blue assay showed that these two compounds were more growth inhibitory in combination than either compound alone (Fig. 6A,C). We then performed median effect analysis to examine whether the higher growth inhibitory rate of the combinations reflected an additive or a synergistic effect. The data showed that all combinations of miR-503 inhibitor and temozolomide were synergistic at the combined concentrations of $5 \mathrm{uM}, 10 \mathrm{uM}, 20 \mathrm{uM}$, and $40 \mathrm{uM}$. These data showed that miR-503 inhibitor augmented the growth inhibitory effect of temozolomide (Fig. 6B,D). The western blot showed that miR-503 inhibitor augmented the level of PARP cleavage, an indicator of apoptosis, when combined with temozolomide. Taken together, these results provide a potential preclinical rationale for examining miR-503 inhibitor in combination with chemotherapy for treatment of glioblastoma.

\section{Discussion}

Glioblastoma multiforme is an aggressive and incurable type of brain cancer associated with a very poor prognosis ${ }^{17}$. Due to its highly invasive nature, it is impossible to completely remove the whole tumor by surgical resection ${ }^{18}$. Over the past decade, there has been a growing interest in the functional role of microRNA in glioblastoma. Based solely on the literature ${ }^{19,20}$, it appears that 256 of the most commonly dysregulated microRNAs are significantly overexpressed in glioblastoma ${ }^{21}$. However, few of these microRNAs, for example microRNA-21, microRNA-17 and microRNA-181 ${ }^{22,23}$, have been thoroughly investigated in regard to their expression or functional role. The functional properties of the majority remain largely unknown. Therefore, there is an urgent need to explore these powerful microRNAs, which could be used as potential targets in glioblastoma therapy.

In this study, we analyzed miRNA expression profiles from a previously published dataset. We found that microRNA-503 is highly upregulated in glioblastoma tissue compared with normal brain tissue, which is consistent with the report from Stefan Wuchty et al. ${ }^{24}$. MicroRNA-503 and microRNA-424 were shown to be regulated by apelin in pulmonary arterial hypertension. However, little was known about how microRNA-503 was upregulated in cancer cells, although there were several reports of its aberrant expression. Jongmin Kim et al. reported that miR-503 and miR-424, which are separated by 250 bp on the X chromosome, are transcribed as a single transcript. Zhang D et al. reported that miR-424 can be induced by TGF- $\beta 1$ in normal fibroblasts. Building on these published data, we found that TGF- $\beta 1$ induces microRNA-503 expression in glioblastoma cells. More elaborately, we performed a ChIP assay to show that smad2/3 binds the microRNA-503 promoter and thus enhances the level of microRNA-503. Furthermore, the core element bound by smad2/3 is highly conserved. It is well established that TGF- $\beta 1$ signaling is dysregulated in glioblastoma. Thus, our data indicates that TGF- $\beta 1$-microRNA-503 may play a key role in glioblastoma progression.

The role of microRNA-503 varies in different cancer tissues. In NSCLC and prostate cancer, microRNA-503 acts as tumor suppressor, however, in esophageal cancer and hepatocellular cancer, microRNA-503 act as an oncogene. In our study of glioblastoma cells, ectopic expression of microRNA-503 with mimics significantly increased cell growth, colony formation and anchorage-independent growth in soft agar. In contrast, knockdown of microRNA-503 sup pressed cell proliferation and anchorage-independent growth. Furthermore, overexpression of microRNA-503 significantly inhibited cell apoptosis. This suggests that microRNA-503 functions as an oncogene and is upregulated in glioblastoma. Moreover, our data showed that PDCD4 is a downstream target of microRNA-503. To translate our findings, we found that inhibition of endogenous microRNA-503 augments the growth inhibitory effect of temozolomide. The median effect analysis showed that microRNA-503 inhibitor and temozolomide at high concentration have a synergistic effect. These results provide a potential preclinical rationale for examining miR-503 inhibitor in combination with chemotherapy for glioblastoma treatment.

In summary, our study shows that microRNA-503 is overexpressed in glioblastoma tissues. Furthermore, TGF- $\beta 1$ induces microRNA-503 at the transcriptional level by binding a core promoter element. We found that microRNA-503 increases proliferation of glioblastoma cells and inhibits apoptosis by directly targeting PDCD4. Finally, miR-503 inhibitor augments the growth inhibitory effect of temozolomide in glioblastoma cells. These results establish miR-503 as a promising molecular target for glioblastoma therapy.

\section{Materials and Methods}

Cell lines and reagent. U251, A172, T98G, LN-229 and 293 T were purchased from ATCC. All the cell lines were grown in Dulbecco's Modified Eagle Medium (DMEM) supplemented with 10\% FBS. The cells were passaged continuously for fewer than 6 months after receipt in our laboratory for relevant studies reported here.

Cell transfection. Cells were transfected with $20 \mathrm{nM}$ miR-503 mimics, miR-503 inhibitor or negative controls (Shanghai GenePharma) using RNAiMAX transfection reagent (Life Technologies) according to the manufacturer's instructions. For plasmid transfections, cells were transfected with Lipofectamine 2000, according to the manufacturer's protocol.

Western blot. The total cell lysates were prepared with RIPA lysis buffer with complete protease inhibitor cocktail. The protein concentration was measured with the BCA Protein Assay Kit. Cell lysates were then subjected to $8 \%$ SDS-PAGE and transferred to a PVDF membrane, and probed with the indicated primary antibodies and secondary antibodies.

Quantitative real-time PCR. Total RNA was extracted with TRIzol reagent. The RNA was subsequently treated with RNase-free DNase I. The cDNA was synthesized with BcaBEST RNA PCR kit from TaKaRa according to the manufacturer's instructions. For detection of miR-503 expression, stem-loop RT-PCR was performed. Quantitative real-time PCR was performed by using SYBR green reagent with ABI Prism 7500 Fast detection 
system. Relative expression was normalized to the expression of U48 small RNA or GAPDH and measured by a comparative CT method.

$3^{\prime}$ UTR luciferase reporter assay and luciferase promoter assay. For the $3^{\prime}$ UTR luciferase reporter assay, the miR-503 mimics or inhibitor and pmirGLO, pmirGLO-PDCD4 3' UTR-wt, pmirGLO-PDCD4-3' UTR-mut were cotransfected into HEK293T. Cell lysates were collected at 48 hours post-transfection. Luciferase activity was measured using the Dual-Luciferase Reporter Assay (Promega). For the luciferase promoter assay, different segments of the miR-503 promoter were produced by PCR using primers and were cloned into pGL3 vectors. The potential binding sites for TGF-b were mutanted for site-specific mutagenesis. U251 cells were transfected with pGL-miR-503-wt-set4, pGL-miR-503-mut-SBE1, pGL-miR-503-mut-SBE2, pGL-miR-503-mut-SBE3, or pGL-miR-503-mut-SBE4 and Renilla. Luciferase activity was measured using the Dual-Luciferase Reporter Assay (Promega).

Chromatin immunoprecipitation assay. 5 million U251 cells were prepared for chromatin immunoprecipitation (ChIP) using the ChIP Assay Kit (Cell Signaling Technology) according to the manufacturer's instructions. The precipitated DNA was analyzed using PCR to amplify five 500 bp regions (set 1 , set 2 , set 3 , set 4 , set 5 ) of the miR-503 promoter. The PCR products were resolved by electrophoresis onto $1 \%$ agarose gel and visualized with ethidium bromide staining.

Cell proliferation assay. For the cell proliferation assay, 5000 cells/well were seeded into 96 well plates. Cell proliferation was determined using AlamarBlue Cell Viability Assay (ThermoFisher Scientific). Data represents the mean + SD of independent experiments.

Soft agar assay. 5000 cells were suspended in a 24-well plate with soft agar and colonies were scored after 4 weeks under a dissection microscope. All experiments were performed three times in triplicate.

Apoptosis assay. Apoptotic cells were detected by flow cytometry using the PE Annexin V apoptosis detection kit (BD Pharmingen) according to the manufacturer's protocol.

In vitro drug treatment and synergism analysis. Cells were seeded in duplicate in 96-well plates 24 hours before exposure to various concentrations of miR-503 inhibitor (The miR-503 inhibitor and temozolomide were used alone at $0.31 \mathrm{uM}, 0.62 \mathrm{uM}, 1.25 \mathrm{uM}, 2.5 \mathrm{uM}, 5 \mathrm{uM}, 10 \mathrm{uM}, 20 \mathrm{uM}, 40 \mathrm{uM}$, and in combination at equipotent concentrations at the same ratios) and temozolomide in constant molar ratios. After 72 hours, Alamar Blue Assay was performed to assess viability. Median effect analysis was performed using Compusyn software. Data was combined from three independent experiments, which were each conducted in duplicate and presented as mean $=$ SEM.

Statistical analysis. Data were analyzed using the two-tailed Student's t test and were shown as mean \pm SE. $\mathrm{P}<0.05$ was considered statistically significant.

\section{References}

1. Omuro, A. \& DeAngelis, L. M. Glioblastoma and other malignant glioblastomas: a clinical review. Jama 310, 1842-1850, https://doi. org/10.1001/jama.2013.280319 (2013).

2. Weller, M. Novel diagnostic and therapeutic approaches to malignant glioblastoma. Swiss medical weekly 141, w13210, https://doi. org/10.4414/smw.2011.13210 (2011).

3. Sturm, D. et al. Paediatric and adult glioblastoma: multiform (epi)genomic culprits emerge. Nature reviews. Cancer 14, 92-107, https://doi.org/10.1038/nrc3655 (2014).

4. Wang, Y. \& Lee, C. G. MicroRNA and cancer-focus on apoptosis. Journal of cellular and molecular medicine 13, 12-23, https://doi. org/10.1111/j.1582-4934.2008.00510.x (2009).

5. Lee, Y. S. \& Dutta, A. MicroRNAs in cancer. Annual review of pathology 4, 199-227, https://doi.org/10.1146/annurev. pathol.4.110807.092222 (2009).

6. Lages, E. et al. MicroRNAs: molecular features and role in cancer. Frontiers in bioscience 17, 2508-2540 (2012).

7. Liu, L., Qu, W. \& Zhong, Z. Down-regulation of miR-503 expression predicate advanced mythological features and poor prognosis in patients with NSCLC. International journal of clinical and experimental pathology 8, 5609-5613 (2015).

8. Guo, J., Liu, X. \& Wang, M. miR-503 suppresses tumor cell proliferation and metastasis by directly targeting RNF31 in prostate cancer. Biochemical and biophysical research communications 464, 1302-1308, https://doi.org/10.1016/j.bbrc.2015.07.127 (2015).

9. Feinmesser, M. et al. Specific MicroRNAs Differentiate Adrenocortical Adenomas from Carcinomas and Correlate With Weiss Histopathologic System. Applied immunohistochemistry \& molecular morphology: AIMM 23, 522-531, https://doi.org/10.1097/ PAI.0000000000000117 (2015).

10. Ide, S. et al. MicroRNA-503 promotes tumor progression and acts as a novel biomarker for prognosis in oesophageal cancer. Anticancer research 35, 1447-1451 (2015).

11. Zhou, J. \& Wang, W. Analysis of microRNA expression profiling identifies microRNA-503 regulates metastatic function in hepatocellular cancer cell. Journal of surgical oncology 104, 278-283, https://doi.org/10.1002/jso.21941 (2011).

12. Held-Feindt, J., Lutjohann, B., Ungefroren, H., Mehdorn, H. M. \& Mentlein, R. Interaction of transforming growth factor-beta (TGF-beta) and epidermal growth factor (EGF) in human glioblastoma cells. Journal of neuro-oncology 63, 117-127 (2003).

13. Stander, M., Naumann, U., Wick, W. \& Weller, M. Transforming growth factor-beta and p-21: multiple molecular targets of decorinmediated suppression of neoplastic growth. Cell and tissue research 296, 221-227 (1999).

14. Kim, J. et al. An endothelial apelin-FGF link mediated by miR-424 and miR-503 is disrupted in pulmonary arterial hypertension. Nature medicine 19, 74-82, https://doi.org/10.1038/nm.3040 (2013).

15. Lankat-Buttgereit, B. \& Goke, R. The tumour suppressor Pdcd4: recent advances in the elucidation of function and regulation. Biology of the cell 101, 309-317, https://doi.org/10.1042/BC20080191 (2009).

16. Arvold, N. D. \& Reardon, D. A. Treatment options and outcomes for glioblastoma in the elderly patient. Clinical interventions in aging 9, 357-367, https://doi.org/10.2147/CIA.S44259 (2014).

17. Seymour, T., Nowak, A. \& Kakulas, F. Targeting Aggressive Cancer Stem Cells in Glioblastoma. Frontiers in oncology 5, 159, https:// doi.org/10.3389/fonc.2015.00159(2015). 
18. Zhang, G. et al. Clinical outcome of gliosarcoma compared with glioblastoma multiforme: a clinical study in Chinese patients. Journal of neuro-oncology 127, 355-362, https://doi.org/10.1007/s11060-015-2046-0 (2016).

19. Szopa, W., Burley, T. A., Kramer-Marek, G. \& Kaspera, W. Diagnostic and Therapeutic Biomarkers in Glioblastoma: Current Status and Future Perspectives. BioMed research international 2017, 8013575, https://doi.org/10.1155/2017/8013575 (2017).

20. Karsy, M. et al. A practical review of prognostic correlations of molecular biomarkers in glioblastoma. Neurosurgical focus 38, E4, https://doi.org/10.3171/2015.1.FOCUS14755 (2015).

21. Shea, A. et al. MicroRNAs in glioblastoma multiforme pathogenesis and therapeutics. Cancer medicine 5, 1917-1946, https://doi. org/10.1002/cam4.775 (2016)

22. Yang, C. H. et al. MicroRNA-21 promotes glioblastoma tumorigenesis by down-regulating insulin-like growth factor-binding protein-3 (IGFBP3). The Journal of biological chemistry 289, 25079-25087, https://doi.org/10.1074/jbc.M114.593863 (2014).

23. Slaby, O. et al. MicroRNA-181 family predicts response to concomitant chemoradiotherapy with temozolomide in glioblastoma patients. Neoplasma 57, 264-269 (2010).

24. Wuchty, S. et al. Prediction of Associations between microRNAs and Gene Expression in Glioblastoma Biology. PloS one 6, e14681, https://doi.org/10.1371/journal.pone.0014681 (2011).

\section{Acknowledgements}

This work was supported by the National Science Foundation of China (81502151, 81502025), and the Natural Science Foundation of Shandong Province (ZR2015HQ008, ZR2015PH011).

\section{Author Contributions}

P.G. and Y.Y. designed the project and performed most of the experiments; H.L., X.D., A.G., S.L., W.L., and L.C., W.Y. performed some of the experiments; Y.Q., L.L., and Y.F. supervised the entire project; P.G. wrote the main manuscript and all authors reviewed the manuscript.

\section{Additional Information}

Supplementary information accompanies this paper at https://doi.org/10.1038/s41598-017-11885-8.

Competing Interests: The authors declare that they have no competing interests.

Publisher's note: Springer Nature remains neutral with regard to jurisdictional claims in published maps and institutional affiliations.

(c) (i) Open Access This article is licensed under a Creative Commons Attribution 4.0 International

License, which permits use, sharing, adaptation, distribution and reproduction in any medium or format, as long as you give appropriate credit to the original author(s) and the source, provide a link to the Creative Commons license, and indicate if changes were made. The images or other third party material in this article are included in the article's Creative Commons license, unless indicated otherwise in a credit line to the material. If material is not included in the article's Creative Commons license and your intended use is not permitted by statutory regulation or exceeds the permitted use, you will need to obtain permission directly from the copyright holder. To view a copy of this license, visit http://creativecommons.org/licenses/by/4.0/.

(C) The Author(s) 2017 